\title{
Conservation laws, symmetry reductions, and exact solutions of some Keller-Segel models
}

\author{
Lihua Zhang $^{1 *}$ and Fengsheng $X \mathrm{u}^{1}$
}

\section{${ }^{*}$ Correspondence:} zzlh100@163.com

'Department of Mathematics, Dezhou University, Dezhou, China

\begin{abstract}
In this paper, three Keller-Segel models are considered from the point of Lie symmetry analysis, conservation laws, symmetry reduction, and exact solutions. By means of Lie symmetry analysis, we first obtain all the symmetries for the three models. Based on the obtained symmetries, many non-trivial and explicit conservation laws for the three models are obtained with the help of Ibragimov's new conservation theorem. Applying the characteristic equations of the obtained symmetries, symmetry reductions and exact solutions are obtained, including solutions expressed by rational functions and Bessel functions.
\end{abstract}

MSC: 76M60; 58J70; 35L65

Keywords: Keller-Segel models; Lie symmetry analysis; Conservation laws; Symmetry reductions

\section{Introduction}

The famous chemotaxis model was proposed by Keller and Segel in the 1970s to describe the aggregation of cellular slime molds Dictyostelium discoideum in response to the chemical cyclic adenosine monophosphate [1, 2]. In its simplified form, Keller-Segel model reads

$$
\left\{\begin{array}{l}
u_{t}=\nabla \cdot(d \nabla u-\delta u \nabla \phi(v)), \\
\varepsilon v_{t}=\alpha \Delta v+f(u, v),
\end{array}\right.
$$

where $u$ and $v$ denote the cell density and chemical concentration, respectively. $d>0$ and $\alpha \geq 0$ are cell and chemical diffusion coefficients, respectively. $\delta>0$ is called the chemotactic coefficient measuring the strength of the chemical signal. Here $\phi(v)$ is referred to as the chemosensitivity function describing the signal detection mechanism and $f(u, v)$ is a function characterizing the chemical growth and degradation. In (1.1), $\varepsilon$ denotes 0 or 1 . When $\varepsilon=1$, (1.1) is called parabolic-parabolic Keller-Segel model; when $\varepsilon=0,(1.1)$ is called elliptic-parabolic Keller-Segel model. Keller-Segel models are used to describe a wide range of processes in biology, ecology, medicine, and so on. The readers are referred to [3-14] for more details about biological motivation and mathematical introduction of Eq. (1.1).

(c) The Author(s) 2018. This article is distributed under the terms of the Creative Commons Attribution 4.0 International License (http://creativecommons.org/licenses/by/4.0/), which permits unrestricted use, distribution, and reproduction in any medium, provided you give appropriate credit to the original author(s) and the source, provide a link to the Creative Commons license, and indicate if changes were made. 
System (1.1) with linear law $\phi(v)=v, \varepsilon=1, f(u, v)=\beta u-\lambda v$ has the form

$$
\left\{\begin{array}{l}
u_{t}=d u_{x x}-\delta\left(u v_{x}\right)_{x} \\
v_{t}=\alpha v_{x x}+\beta u-\lambda v
\end{array}\right.
$$

and is also called the minimum chemotaxis model, see a review article [6]. When $d=\delta=1$, $\lambda=0$, system (1.2) becomes of the form in $[8,9]$ :

$$
\left\{\begin{array}{l}
u_{t}=u_{x x}-\left(u v_{x}\right)_{x} \\
v_{t}=\alpha v_{x x}+\beta u
\end{array}\right.
$$

For the integrable case $\alpha=2$, an exact solution of (1.3) has been derived in [8], more exact solutions of (1.3) have been derived in [9].

System (1.1) with logarithmic sensitivity $\phi(v)=\ln v, \varepsilon=1, f(u, v)=\beta u-\lambda v$ is expressed by

$$
\left\{\begin{array}{l}
u_{t}=d u_{x x}-\delta\left(\frac{u v_{x}}{v}\right)_{x} \\
v_{t}=\alpha v_{x x}+\beta u-\lambda v
\end{array}\right.
$$

and has prominent specific applications. For the special case $\alpha=0, \lambda=0$ of (1.4), Keller and Segel [10] performed theoretical analysis to interpret the propagating travelling bands of bacterial chemotaxis experimentally observed in $[11,12]$.

It is known that Lie symmetry analysis is a powerful and systematic method for dealing with partial differential equations (PDEs) [15-21]. Lie symmetry analysis has also been extended to fractional partial differential equations (FPDEs) in recent years [22-25]. Moreover, this method has had a profound impact on both pure and applied areas of mathematics, physics, mechanics, etc. Based on the symmetries of a PDE or a FPDE, many other important properties of the equation such as integrability, conservation laws, reduction equations, and exact solutions can be considered successively [18-25]. For the special case $\phi(v)=v, \varepsilon=0, f(u, v)=\beta u-\lambda v$, Lie symmetry analysis and self-similar solutions are considered in [26], and a natural continuation of [26] for a $(1+2)$-dimensional Keller-Segel model is considered in $[27,28]$. As far as we know, Lie symmetry analysis and conservation laws of (1.2), (1.3), and (1.4) have not been studied. To provide more information for understanding the three models, we will study symmetry, conservation laws, symmetry reductions, and exact solutions of (1.2), (1.3), and (1.4).

The rest of the paper is organized as follows. In Sect. 2, Lie symmetry analysis of the three models is performed. In Sect. 3, formal Lagrangian and adjoint systems of the three models are derived to obtain the conservation laws. In Sect. 4, non-trivial and explicit conservation laws are derived. In Sect. 5, we use the symmetry to get symmetry reductions and new exact solutions of the three models. The last section is a short summary and discussion.

\section{Symmetry analysis of (1.2), (1.3), and (1.4)}

Recall that the geometric vector field of system (1.1) is as follows:

$$
V=\xi(x, t, u, v) \frac{\partial}{\partial x}+\tau(x, t, u, v) \frac{\partial}{\partial t}+\phi(x, t, u, v) \frac{\partial}{\partial u}+\psi(x, t, u, v) \frac{\partial}{\partial v}
$$


where the coefficient functions $\xi(x, t, u, v), \tau(x, t, u, v), \phi(x, t, u, v)$, and $\psi(x, t, u, v)$ are to be determined later. Equation (2.1) is also called a symmetry of (1.1).

If the vector field (2.1) generates a symmetry of the three systems (1.2), (1.3), and (1.4), then $V$ must satisfy the Lie symmetry condition

$$
\left\{\begin{array}{l}
\left.\operatorname{pr}^{(m)} V\left(\Delta_{1}\right)\right|_{\Delta_{1}=0}=0 \\
\left.\operatorname{pr}^{(n)} V\left(\Delta_{2}\right)\right|_{\Delta_{2}=0}=0
\end{array}\right.
$$

where $\operatorname{pr}^{(m)} V$ denotes the $m$ th prolongation of $V$, and $\Delta_{1}=u_{t}-d u_{x x}+\delta\left(u v_{x}\right)_{x}, \Delta_{2}=v_{t}-$ $\alpha v_{x x}-\beta u+\lambda v$ for system (1.2), $\Delta_{1}=u_{t}-u_{x x}+\left(u v_{x}\right)_{x}, \Delta_{2}=v_{t}-\alpha v_{x x}-\beta u$ for system (1.3), $\Delta_{1}=u_{t}-d u_{x x}+\delta\left(\frac{u v_{x}}{v}\right)_{x}, \Delta_{2}=v_{t}-\alpha v_{x x}-\beta u+\lambda v$ for system (1.4), respectively. For example, in view of system (1.2), we have

$$
\operatorname{pr}^{(2)} V=\phi \frac{\partial}{\partial u}+\psi \frac{\partial}{\partial v}+\phi^{t} \frac{\partial}{\partial u_{t}}+\psi^{t} \frac{\partial}{\partial v_{t}}+\phi^{x} \frac{\partial}{\partial u_{x}}+\psi^{x} \frac{\partial}{\partial v_{x}}+\phi^{x x} \frac{\partial}{\partial u_{x x}}+\psi^{x x} \frac{\partial}{\partial v_{x x}}
$$

where the coefficient functions are given by

$$
\begin{aligned}
& \phi^{x}=D_{x}\left(\phi-\xi u_{x}-\tau u_{t}\right)+\xi u_{x x}+\tau u_{x t}, \\
& \phi^{t}=D_{t}\left(\phi-\xi u_{x}-\tau u_{t}\right)+\xi u_{x t}+\tau u_{t t}, \\
& \phi^{x x}=D_{x x}\left(\phi-\xi u_{x}-\tau u_{t}\right)+\xi u_{x x x}+\tau u_{t x x}, \\
& \psi^{x}=D_{x}\left(\psi-\xi v_{x}-\tau v_{t}\right)+\xi v_{x x}+\tau v_{x t}, \\
& \psi^{t}=D_{t}\left(\psi-\xi v_{x}-\tau v_{t}\right)+\xi v_{x t}+\tau v_{t t}, \\
& \psi^{x x}=D_{x x}\left(\psi-\xi v_{x}-\tau v_{t}\right)+\xi v_{x x x}+\tau v_{x x t},
\end{aligned}
$$

$D_{x}$ and $D_{t}$ are total differential operators with respect to $x$ and $t$, respectively. Then, in terms of the Lie symmetry analysis method, we can obtain all of the geometric vector fields of the three systems.

(i) For system (1.2), the vector field for an arbitrary value of $\lambda$ is

$$
V_{1}=\frac{\partial}{\partial x}, \quad V_{2}=\frac{\partial}{\partial t}, \quad V_{3}=e^{-\lambda t} \frac{\partial}{\partial v} .
$$

When $\lambda=0$, in addition to (2.4), there is another vector

$$
V_{4}=\frac{x}{2} \frac{\partial}{\partial x}+t \frac{\partial}{\partial t}-u \frac{\partial}{\partial u}
$$

(ii) For system (1.3), the vector field is

$$
V_{1}=\frac{\partial}{\partial x}, \quad V_{2}=\frac{\partial}{\partial t}, \quad V_{3}=\frac{\partial}{\partial v}, \quad V_{4}=\frac{x}{2} \frac{\partial}{\partial x}+t \frac{\partial}{\partial t}-u \frac{\partial}{\partial u} .
$$

(iii) For system (1.4), the vector field for an arbitrary value of $\lambda$ is

$$
V_{1}=\frac{\partial}{\partial x}, \quad V_{2}=\frac{\partial}{\partial t}, \quad V_{3}=u \frac{\partial}{\partial u}+v \frac{\partial}{\partial v} .
$$


When $\lambda=0$, in addition to (2.6), there is another vector

$$
V_{4}=\frac{x}{2} \frac{\partial}{\partial x}+t \frac{\partial}{\partial t}-u \frac{\partial}{\partial u}
$$

Moreover, it is necessary to show that the vector fields of the systems are closed under the Lie racket, respectively. Taking (2.5) for an example, we have

$$
\begin{array}{lll}
{\left[V_{1}, V_{2}\right]=0,} & {\left[V_{1}, V_{3}\right]=0,} & {\left[V_{1}, V_{4}\right]=\frac{1}{2} V_{1},} \\
{\left[V_{2}, V_{3}\right]=0,} & {\left[V_{2}, V_{4}\right]=V_{2},} & {\left[V_{3}, V_{4}\right]=0 .}
\end{array}
$$

So, the vector field of (1.3) is spanned by a four-dimensional Lie algebra. In fact, the vector fields of (1.2) and (1.4) are also spanned by finite dimensional Lie algebra.

\section{Formal Lagrangians and adjoint systems}

The construction of explicit forms of conservation laws plays an important role in the study of nonlinear science, as they are used for the development of appropriate numerical methods and for mathematical analysis, in particular, existence, uniqueness, and stability analysis [29-31]. In addition, the existence of a large number of conservation laws of a partial differential equation (system) is a strong indication of its integrability. There are many methods to get the conservation laws of differential equations, such as Noether's theorem [32], the partial Noether approach [33], and so on [34-38]. Among those, the new conservation theorem given by Ibragimov is one of the most frequently used methods since it does not require the existence of classical Lagrangians and can be used to find conservation laws associated with the known Lie, Lie-Bäcklund, or non-local symmetries $[34,39]$.

\subsection{A general theorem on conservation laws}

To derive conservation laws of systems (1.2), (1.3), and (1.4), we use the following new conservation theorem proved in $[32,33]$.

Theorem 1 Every Lie point, Lie-Bäcklund, and non-local symmetry

$$
X=\xi^{i}\left(x, u, u_{(1)}, \ldots\right) \frac{\partial}{\partial x^{i}}+\eta^{s}\left(x, u, u_{(1)}, \ldots\right) \frac{\partial}{\partial u^{s}}
$$

of a system of $m$ equations

$$
F_{s}\left(x, u, u_{(1)}, \ldots, u_{(N)}\right)=0, \quad s=1, \ldots, m,
$$

with $n$ independent variables $x=\left(x^{1}, \ldots, x^{n}\right)$ and $m$ dependent variables $u=\left(u^{1}, \ldots, u^{m}\right)$ provides a conservation law for system (3.1) and the corresponding adjoint system

$$
F_{s}^{*}\left(x, u, v, u_{(1)}, v_{(1)}, \ldots, u_{(N)}, v_{(N)}\right) \equiv \frac{\delta\left(v^{i} F_{i}\right)}{\delta u^{s}}=0, \quad s=1, \ldots, m
$$


Then the elements of the conservation vector $T=\left(T^{1}, \ldots, T^{n}\right)$ are defined by the following expression:

$$
\begin{aligned}
T^{i}= & \xi^{i} L+W^{s}\left[\frac{\partial L}{\partial u_{i}^{s}}-D_{x^{j}}\left(\frac{\partial L}{\partial u_{i j}^{s}}\right)+D_{x^{j}} D_{x^{k}}\left(\frac{\partial L}{\partial u_{i j k}^{s}}\right)-\cdots\right] \\
& +D_{x j}\left(W^{s}\right)\left[\frac{\partial L}{\partial u_{i j}^{s}}-D_{x^{k}}\left(\frac{\partial L}{\partial u_{i j k}^{s}}\right)+D_{x^{k}} D_{x^{r}}\left(\frac{\partial L}{\partial u_{i j k r}^{s}}\right)-\cdots\right] \\
& +D_{x^{j}} D_{x^{k}}\left(W^{s}\right)\left[\frac{\partial L}{\partial u_{i j k}^{s}}-D_{x^{r}}\left(\frac{\partial L}{\partial u_{i j k r}^{s}}\right)+\cdots\right]+\cdots
\end{aligned}
$$

with

$$
W^{s}=\eta^{s}-\xi^{i} u_{i}^{s}, \quad s=1, \ldots, m .
$$

\subsection{Formal Lagrangians and adjoint systems}

According to the method of constructing Lagrangians in [32,33], the formal Lagrangian of system (1.2) is

$$
L=Z \Delta_{1}+\omega \Delta_{2}=Z\left(u_{t}-d u_{x x}+\delta u_{x} v_{x}+\delta u v_{x x}\right)+\omega\left(v_{t}-\alpha v_{x x}-\beta u+\lambda v\right),
$$

where $Z$ and $\omega$ are two new dependent variables with respect to $x$ and $t$. Following the idea in [32, 33], the adjoint system of system (1.2) is

$$
\left\{\begin{array}{l}
\Delta_{1}^{*}=\frac{\delta L}{\delta u}=0 \\
\Delta_{2}^{*}=\frac{\delta L}{\delta v}=0
\end{array}\right.
$$

where $\frac{\delta}{\delta u}$ and $\frac{\delta}{\delta v}$ are Euler operators,

$$
\begin{aligned}
& \frac{\delta}{\delta u}=\frac{\partial}{\partial u}-D_{x} \frac{\partial}{\partial u_{x}}-D_{t} \frac{\partial}{\partial u_{t}}+D_{x x} \frac{\partial}{\partial u_{x x}} \\
& \frac{\delta}{\delta v}=\frac{\partial}{\partial v}-D_{x} \frac{\partial}{\partial v_{x}}-D_{t} \frac{\partial}{\partial v_{t}}+D_{x x} \frac{\partial}{\partial v_{x x}}
\end{aligned}
$$

Substituting (3.5) and (3.7) into system (3.6), the adjoint system for system (1.2) is expressed as follows:

$$
\left\{\begin{array}{l}
\Delta_{1}^{*} \equiv Z_{t}+d z_{x x}+\delta Z_{x} v_{x}+\beta \omega=0 \\
\Delta_{2}^{*} \equiv \omega_{t}+\alpha \omega_{x x}-\delta Z_{x x} u-\delta Z_{x} u_{x}-\lambda \omega=0
\end{array}\right.
$$

The formal Lagrangian of system (1.3) is

$$
L=Z \Delta_{1}+\omega \Delta_{2}=Z\left(u_{t}-u_{x x}+u_{x} v_{x}+u v_{x x}\right)+\omega\left(v_{t}-\alpha v_{x x}-\beta u\right),
$$

and the adjoint system for system (1.3) is

$$
\left\{\begin{array}{l}
\Delta_{1}^{*} \equiv Z_{t}+z_{x x}+Z_{x} v_{x}+\beta \omega=0 \\
\Delta_{2}^{*} \equiv \omega_{t}+\alpha \omega_{x x}-Z_{x x} u-Z_{x} u_{x}=0
\end{array}\right.
$$


In a similar way with system (1.2), the formal Lagrangian of system (1.4) is

$$
\begin{aligned}
L & =Z \Delta_{1}+\omega \Delta_{2} \\
& =Z\left(u_{t}-d u_{x x}+\delta \frac{u_{x} v_{x}}{v}+\delta \frac{u v_{x x}}{v}-\delta \frac{u v_{x}^{2}}{v^{2}}\right)+\omega\left(v_{t}-\alpha v_{x x}-\beta u+\lambda v\right),
\end{aligned}
$$

and the adjoint system for system (1.4) is

$$
\left\{\begin{array}{l}
\Delta_{1}^{*} \equiv Z_{t}+d Z_{x x}+\delta \frac{Z_{x} v_{x}}{v}+\beta \omega=0, \\
\Delta_{2}^{*} \equiv \omega_{t}+\alpha \omega_{x x}-\delta \frac{Z_{x} u_{x}}{v}-\delta \frac{Z_{x x} u}{v}-\lambda \omega=0 .
\end{array}\right.
$$

\section{Conservation laws for systems (1.2), (1.3), and (1.4)}

\subsection{Conservation laws for system (1.2)}

There are three symmetries for system (1.2). Based on Theorem 1, there is a conservation law corresponding to every symmetry. For Lie symmetry $V_{1}=\frac{\partial}{\partial x}$, the characteristic functions are $W^{1}=-u_{x}, W^{2}=-v_{x}$, and the conservation law of system (1.2) and adjoint system (3.8) derived by $V_{1}$ is

$$
\begin{aligned}
& X^{1}=Z u_{t}+\omega v_{t}-\beta \omega u+\lambda \omega v-d u_{x} Z_{x}+\delta u v_{x} z_{x}-\alpha v_{x} \omega_{x}, \\
& T^{1}=-Z u_{x}-\omega v_{x} .
\end{aligned}
$$

For Lie symmetry $V_{2}=\frac{\partial}{\partial t}$, the characteristic functions are $W^{1}=-u_{t}, W^{2}=-v_{t}$, and the conservation law of system (1.2) and adjoint system (3.8) derived by $V_{2}$ is

$$
\begin{aligned}
& X^{2}=-\delta Z u_{t} v_{x}-d u_{t} Z_{x}+\delta u v_{t} Z_{x}-\alpha v_{t} \omega_{x}+d Z u_{x t}-\delta Z u v_{x t}+\alpha \omega v_{x t}, \\
& T^{2}=-d Z u_{x x}+\delta Z u_{x} v_{x}+\delta Z u v_{x x}-\alpha \omega v_{x x}-\beta \omega u+\lambda \omega \nu
\end{aligned}
$$

For Lie symmetry $V_{3}=e^{-\lambda t} \frac{\partial}{\partial v}$, the characteristic functions are $W^{1}=0, W^{2}=e^{-\lambda t}$, and the conservation law of system (1.2) and adjoint system (3.8) derived by $V_{3}$ is

$$
\begin{aligned}
X^{3} & =\frac{1}{e^{\lambda t}}\left(-\delta u Z_{x}+\alpha \omega_{x}\right), \\
T^{3} & =\frac{1}{e^{\lambda t}} \omega .
\end{aligned}
$$

In the above expressions of the conservation laws of systems (1.2) and (3.8), $Z$ and $\omega$ are arbitrary solutions of system (3.8). If we can find exact solutions of (3.8), explicit conservation laws of (1.2) can be obtained by substituting them to the above expressions. For example,

$$
\begin{aligned}
& Z=C_{1}+C_{2} e^{\lambda t}, \\
& \omega=-\frac{\lambda C_{2}}{\beta} e^{\lambda t},
\end{aligned}
$$


where $C_{1}$ and $C_{2}$ are nonzero constants, is a solution of (3.8). By substituting it to (4.2), we can obtain an explicit conservation law of (1.2) corresponding to symmetry $V_{2}$ :

$$
\begin{aligned}
X^{2}= & -\frac{1}{\beta}\left(\delta C_{1} \beta u_{t} v_{x}+\delta C_{2} \beta e^{\lambda t} u_{t} v_{x}-d C_{1} \beta u_{x t}-d C_{2} \beta e^{\lambda t} u_{x t}\right. \\
& \left.+\delta C_{1} \beta u v_{x t}+\delta C_{2} \beta e^{\lambda t} u v_{x t}+\alpha C_{2} \lambda e^{\lambda t} v_{x t}\right), \\
T^{2}= & \frac{1}{\beta}\left(-d C_{1} \beta u_{x x}-d C_{2} \beta e^{\lambda t} u_{x x}+\delta C_{1} \beta u_{x} v_{x}+\delta C_{2} \beta e^{\lambda t} u_{x} v_{x}+\delta C_{1} \beta u v_{x x}\right. \\
& \left.+\delta C_{2} \beta e^{\lambda t} u v_{x x}+\alpha C_{2} \lambda e^{\lambda t} v_{x x}+\lambda \beta C_{2} e^{\lambda t} u-\lambda^{2} C_{2} e^{\lambda t} v\right) .
\end{aligned}
$$

\subsection{Conservation laws for system (1.3)}

There are four symmetries for system (1.3). Next we will study a conservation law for the system consisting of (1.3) and (3.10) according to Theorem 1. For Lie symmetry $V_{1}=\frac{\partial}{\partial x}$, the characteristic functions are $W^{1}=-u_{x}, W^{2}=-v_{x}$, and the conservation law of system (1.3) and its adjoint system (3.10) derived by $V_{1}$ is

$$
\begin{aligned}
& X^{1}=Z u_{t}+\omega v_{t}-\beta \omega u-u_{x} Z_{x}+u v_{x} z_{x}-\alpha v_{x} \omega_{x}, \\
& T^{1}=-Z u_{x}-\omega v_{x} .
\end{aligned}
$$

For Lie symmetry $V_{2}=\frac{\partial}{\partial t}$, the characteristic functions are $W^{1}=-u_{t}, W^{2}=-v_{t}$, and the conservation law of system (1.3) and its adjoint system (3.10) derived by $V_{2}$ is

$$
\begin{aligned}
& X^{2}=-Z u_{t} v_{x}-u_{t} Z_{x}+u v_{t} Z_{x}-\alpha v_{t} \omega_{x}+Z u_{x t}-Z u v_{x t}+\alpha \omega v_{x t}, \\
& T^{2}=-Z u_{x x}+Z u_{x} v_{x}+Z u v_{x x}-\alpha \omega v_{x x}-\beta \omega u .
\end{aligned}
$$

For Lie symmetry $V_{3}=\frac{\partial}{\partial v}$, the characteristic functions are $W^{1}=0, W^{2}=1$, and the conservation law of system (1.3) and its adjoint system (3.10) derived by $V_{3}$ is

$$
\begin{aligned}
& X^{3}=-Z_{x} u+\alpha \omega_{x}, \\
& T^{3}=\omega .
\end{aligned}
$$

For Lie symmetry $V_{4}=\frac{x}{2} \frac{\partial}{\partial x}+t \frac{\partial}{\partial t}-u \frac{\partial}{\partial u}$, the characteristic functions are $W^{1}=-u-\frac{x}{2} u_{x}-$ $t u_{t}, W^{2}=-\frac{x}{2} v_{x}-t v_{t}$, and the conservation law of system (1.3) and its adjoint system (3.10) derived by $V_{4}$ is

$$
\begin{aligned}
X^{4}= & -u Z_{x}+\frac{x}{2} \omega v_{t}+\frac{\alpha}{2} \omega v_{x}+\frac{3}{2} Z u_{x}+\frac{x}{2} Z u_{t}-\frac{\beta}{2} x \omega u-\frac{3}{2} u Z v_{x} \\
& -\frac{1}{2} x u_{x} Z_{x}-t u_{t} z_{x}-\frac{\alpha}{2} x \omega_{x} v_{x}-t \alpha v_{t} \omega_{x}+t Z u_{x t}+t \alpha \omega v_{x t}-t z u_{t} v_{x} \\
& +\frac{1}{2} x u v_{x} Z_{x}+t u v_{t} Z_{x}-t u Z v_{x t}, \\
T^{4}= & -t Z u_{x x}+t Z u_{x} v_{x}+t Z u v_{x x}-t \alpha \omega v_{x x}-t \beta \omega u-Z u-\frac{1}{2} x Z u_{x}-\frac{1}{2} x \omega v_{x} .
\end{aligned}
$$

In the above expressions of the conservation laws of systems (1.3) and (3.10), $Z$ and $\omega$ are arbitrary solutions of system (3.10). If we can find exact solutions of (3.10), explicit 
conservation laws of (1.3) can be obtained by substituting them to the above expressions. For example,

$$
\begin{aligned}
& Z=t, \\
& \omega=-\frac{1}{\beta},
\end{aligned}
$$

is a solution of (3.10). By substituting it to (4.7), we can obtain an explicit conservation law of (1.3) corresponding to symmetry $V_{4}$ :

$$
\begin{aligned}
X^{4}= & -\frac{\alpha}{2 \beta} v_{x}+\frac{x}{2} t u_{t}+t^{2} u_{x t}-\frac{3}{2} t u v_{x}-\frac{x}{2 \beta} v_{t}+\frac{1}{2} x u-t^{2} u_{t} v_{x} \\
& -t^{2} u v_{x t}-\frac{\alpha}{\beta} t v_{x t}+\frac{3}{2} t u_{x}, \\
T^{4}= & -t^{2} u_{x x}+t^{2} u_{x} v_{x}+t^{2} u v_{x x}+t \frac{\alpha}{\beta} v_{x x}-\frac{1}{2} x t u_{x}+\frac{1}{2 \beta} x v_{x} .
\end{aligned}
$$

\subsection{Conservation laws for system (1.4)}

There are three symmetries for system (1.4) with an arbitrary value of $\lambda$. Next we will study a conservation law for the system consisting of (1.4) and (3.12) according to Theorem 1. For Lie symmetry $V_{1}=\frac{\partial}{\partial x}$, the characteristic functions are $W^{1}=-u_{x}, W^{2}=-v_{x}$, and the conservation law of system (1.4) and its adjoint system (3.12) derived by $V_{1}$ is

$$
\begin{aligned}
& X^{1}=Z u_{t}+\omega v_{t}-\beta \omega u+\lambda \omega v-d u_{x} Z_{x}+\frac{\delta u}{v} v_{x} z_{x}-\alpha v_{x} \omega_{x}, \\
& T^{1}=-Z u_{x}-\omega v_{x} .
\end{aligned}
$$

For Lie symmetry $V_{2}=\frac{\partial}{\partial t}$, the characteristic functions are $W^{1}=-u_{t}, W^{2}=-v_{t}$, and the conservation law of system (1.4) and its adjoint system (3.12) derived by $V_{2}$ is

$$
\begin{aligned}
X^{2}= & -\frac{\delta Z}{v} u_{t} v_{x}-d u_{t} Z_{x}+\frac{\delta Z u}{v^{2}} v_{t} v_{x}+\frac{\delta u}{v} v_{t} Z_{x}-\alpha v_{t} \omega_{x} \\
& +d Z u_{x t}-\frac{\delta Z u}{v} v_{x t}+\alpha \omega v_{x t}, \\
T^{2}= & -d Z u_{x x}+\frac{\delta Z}{v} u_{x} v_{x}+\frac{\delta Z}{v} u v_{x x}-\frac{\delta Z u}{v^{2}} v_{x}^{2}-\alpha \omega v_{x x}-\beta \omega u+\lambda \omega v .
\end{aligned}
$$

For Lie symmetry $V_{3}=u \frac{\partial}{\partial u}+v \frac{\partial}{\partial v}$, the characteristic functions are $W^{1}=u, W^{2}=v$, and the conservation law of system (1.4) and its adjoint system (3.12) derived by $V_{3}$ is

$$
\begin{aligned}
X^{3} & =\frac{\delta Z u}{v} v_{x}+d u Z_{x}-\delta u Z_{x}+\alpha \omega_{x} v-d Z u_{x}-\alpha \omega v_{x}, \\
T^{3} & =u Z+\omega v .
\end{aligned}
$$

For the case $\lambda=0$, there is another symmetry $V_{4}=\frac{x}{2} \frac{\partial}{\partial x}+t \frac{\partial}{\partial t}-u \frac{\partial}{\partial u}$, the characteristic functions are $W^{1}=-u-\frac{x}{2} u_{x}-t u_{t}, W^{2}=-\frac{x}{2} v_{x}-t v_{t}$, and the conservation law of system 
(1.4) and its adjoint system (3.12) with $\lambda=0$ derived by $V_{4}$ is

$$
\begin{aligned}
X^{4}= & \frac{3 d}{2} Z u_{x}-\alpha t \omega_{x} v_{t}-\frac{\alpha}{2} x v_{x} \omega_{x}-d t u_{t} Z_{x}-\frac{d}{2} x Z_{x} u_{x}+d t Z u_{x t}+\frac{1}{2} x Z u_{t} \\
& +\alpha t \omega v_{x t}-\frac{\delta t}{v} Z u v_{x t}+\frac{\delta t}{v} u v_{t} Z_{x}+\frac{\delta x}{2 v} u v_{x} Z_{x}-\frac{\delta t}{v} Z u_{t} v_{x}+\frac{1}{2} x \omega v_{t} \\
& -\frac{\beta}{2} x \omega u-\frac{3 \delta}{2 v} Z u v_{x}-d u Z_{x}+\frac{\alpha}{2} \omega v_{x}+\frac{\delta t}{v^{2}} Z u v_{t} v_{x}, \\
T^{4}= & -d t Z u_{x x}+\frac{\delta t}{v} Z u_{x} v_{x}+\frac{\delta t}{v} Z u v_{x x}-\frac{\delta t}{v^{2}} Z u v_{x}^{2}-\alpha t \omega v_{x x} \\
& -\beta t \omega u-Z u-\frac{1}{2} x Z u_{x}-\frac{1}{2} x \omega v_{x} .
\end{aligned}
$$

In the above expressions of the conservation laws of systems (1.4) and (3.12), $Z$ and $\omega$ are arbitrary solutions of system (3.12). If we can find exact solutions of (3.12), explicit conservation laws of (1.4) can be obtained by substituting them to the above expressions. For example,

$$
\begin{aligned}
& Z=e^{\lambda t}, \\
& \omega=-\frac{\lambda}{\beta} e^{\lambda t},
\end{aligned}
$$

is a solution of (3.12). By substituting it to (4.11), we can obtain an explicit conservation law of (1.4) corresponding to symmetry $V_{3}$ :

$$
\begin{aligned}
& X^{3}=-\frac{e^{\lambda t}}{\beta v}\left(-\beta \delta u v_{x}+\beta d u_{x} v-\lambda \alpha \nu v_{x}\right), \\
& T^{3}=-\frac{e^{\lambda t}}{\beta}(-\beta u+\lambda v) .
\end{aligned}
$$

Remark 1 Here we should point out that all the conservation laws obtained in this section are non-trivial and have been checked by Maple software.

\section{Symmetry reductions and exact solutions}

In Sect. 2, we have obtained the Lie symmetries of (1.2), (1.3), and (1.4). In this section, we will investigate the symmetry reductions and exact solutions for the three equations. Since (1.3) is a special case of (1.2), and its solutions have been studied in [9], we mainly focus on the reductions of (1.2) and (1.4). Using the obtained symmetries (2.1), similarity variables and symmetry reductions can be found by solving the corresponding characteristic equation

$$
\frac{d x}{\xi(x, t, u, v)}=\frac{d t}{\tau(x, t, u, v)}=\frac{d u}{\phi(x, t, u, v)}=\frac{d v}{\psi(x, t, u, v)}
$$

\subsection{Symmetry reductions and exact solutions of (1.2)}

For Lie symmetry $V_{1}=\frac{\partial}{\partial x}$, we can obtain

$$
\begin{aligned}
& u=u(t), \\
& v=v(t)
\end{aligned}
$$


Substituting (5.1) to (1.2), we can obtain the exact solutions of (1.2) as follows:

$$
\begin{aligned}
& u=M_{2}, \\
& v=\frac{\beta}{\lambda} M_{2}+M_{1} e^{-\lambda t},
\end{aligned}
$$

where $M_{1}$ and $M_{2}$ are arbitrary constants.

For Lie symmetry $V_{2}=\frac{\partial}{\partial t}$, we can obtain

$$
\begin{aligned}
& u=u(x), \\
& v=v(x) .
\end{aligned}
$$

Substituting (5.3) to (1.2), we can obtain the following reduction equations:

$$
\begin{aligned}
& -d u_{x x}+\delta\left(u v_{x}\right)_{x}=0, \\
& u=-\frac{1}{\beta}\left(\alpha v_{x x}-\lambda v\right) .
\end{aligned}
$$

Taking the second equation of (5.4) into the first equation, after simplification we can get

$$
d \alpha v_{x x}-d \lambda v-\frac{1}{2} \delta \alpha v_{x}^{2}+\frac{1}{2} \delta \lambda v^{2}=0
$$

so an exact solution of (1.2) can be found by solving (5.5).

For Lie symmetry $V_{3}=e^{-\lambda t} \frac{\partial}{\partial v}$, we can obtain

$$
\begin{aligned}
& u=u(x, t), \\
& v=M_{0} .
\end{aligned}
$$

Substituting (5.6) to (1.2), we can obtain the following solutions:

$$
\begin{aligned}
& u=\frac{\lambda}{\beta} M_{0}, \\
& v=M_{0} .
\end{aligned}
$$

From the symmetry $V_{4}=\frac{x}{2} \frac{\partial}{\partial x}+t \frac{\partial}{\partial t}-u \frac{\partial}{\partial u}$ for the special case $\lambda=0$, we can obtain

$$
\begin{aligned}
& u=\frac{1}{t} F, \\
& v=G,
\end{aligned}
$$

where $F=F(\theta), G=G(\theta), \theta=\frac{x^{2}}{t}$. Substituting (5.8) to (1.2), we can obtain the following reduction equations:

$$
\begin{aligned}
& -F-\theta F^{\prime}-4 d \theta F^{\prime \prime}-2 d F^{\prime}+2 \delta F G^{\prime}+4 \delta \theta\left(F G^{\prime}\right)^{\prime}=0, \\
& -\theta G^{\prime}-4 \alpha \theta G^{\prime \prime}-2 \alpha G^{\prime}-\beta F=0 .
\end{aligned}
$$

If we can find solutions of (5.9), new solutions of (1.3) can also be obtained. 
To compare with the result for (1.3) in [9], we only consider the symmetry $c V_{1}+V_{2}=$ $c \frac{\partial}{\partial x}+\frac{\partial}{\partial t}$, where $c$ is a constant. From the corresponding characteristic equation $\frac{d x}{c}=\frac{d t}{1}$, we can obtain

$$
\begin{aligned}
& u=u(\theta), \\
& v=v(\theta),
\end{aligned}
$$

where $\theta=x-c t$. Substituting (5.10) to (1.3), we can obtain the following reduction equations:

$$
\begin{aligned}
& c u_{\theta}+u_{\theta \theta}-\left(u_{\theta} v_{\theta}+u v_{\theta \theta}\right)=0, \\
& u=-\frac{1}{\beta}\left(c v_{\theta}+\alpha v_{\theta \theta}\right) .
\end{aligned}
$$

The first equation of (5.11) can be integrated once and becomes

$$
c u+u_{\theta}-u v_{\theta}+N=0
$$

where $N$ is an arbitrary constant. Taking the second equation of (5.11) into (5.12), one can obtain

$$
-\alpha v_{\theta \theta \theta}-c \alpha v_{\theta \theta}-c v_{\theta \theta}-c^{2} v_{\theta}+\alpha v_{\theta} v_{\theta \theta}+c v_{\theta}^{2}+N=0
$$

Equation (5.13) is exactly the same as (5) in [9] and a lot of traveling solutions have been found.

\subsection{Symmetry reductions and exact solutions of (1.4)}

For Lie symmetry $V_{1}=\frac{\partial}{\partial x}$, we can obtain

$$
\begin{aligned}
& u=u(t), \\
& v=v(t) .
\end{aligned}
$$

Substituting (5.14) to (1.4), we can obtain the exact solutions of (1.4) as follows:

$$
\begin{aligned}
& u=M_{2}, \\
& v=\frac{\beta}{\lambda} M_{2}+M_{1} e^{-\lambda t},
\end{aligned}
$$

where $M_{1}$ and $M_{2}$ are arbitrary constants.

For Lie symmetry $V_{2}=\frac{\partial}{\partial t}$, we can obtain

$$
\begin{aligned}
& u=u(x), \\
& v=v(x) .
\end{aligned}
$$


Substituting (5.16) to (1.4), we can obtain the following reduction equations:

$$
\begin{aligned}
& -d u_{x x}+\delta\left(\frac{u v_{x}}{v}\right)_{x}=0 \\
& u=-\frac{-\alpha v_{x x}+\lambda v}{\beta}
\end{aligned}
$$

When $d=\delta$, we can find a solution for (1.4) as follows:

$$
\begin{aligned}
& u=\frac{\alpha-\lambda M_{1}^{2}}{2 \beta M_{1}^{2}}\left(e^{\frac{2\left(x+M_{2}\right)}{M_{1}}}+1\right) \sqrt{-M_{3} e^{\frac{2\left(x+M_{2}\right)}{-M_{1}}}}, \\
& v=-\frac{1}{2} \sqrt{-M_{3} e^{\frac{2\left(x+M_{2}\right)}{-M_{1}}}}\left(e^{\frac{2\left(x+M_{2}\right)}{M_{1}}}+1\right),
\end{aligned}
$$

where $M_{1}, M_{2}$, and $M_{3}$ are constants, $M_{1} \neq 0, M_{3}<0$.

For Lie symmetry $V_{3}=u \frac{\partial}{\partial u}+v \frac{\partial}{\partial v}$, we can obtain

$$
\begin{aligned}
& u=v F, \\
& v=v,
\end{aligned}
$$

where $F=F(x, t)$. Substituting (5.19) to (1.4), we can obtain the following reduction equations:

$$
\begin{aligned}
& v_{t} F+\nu F_{t}+(\delta-d) \nu_{x x} F+(\delta-2 d) \nu_{x} F_{x}-d \nu F_{x x}=0 \\
& v_{t}-\alpha v_{x x}-\beta \nu F+\lambda \nu=0 .
\end{aligned}
$$

To solve (5.20) is difficult, so we set

$$
\begin{aligned}
& F=F(\theta), \\
& v=v(\theta),
\end{aligned}
$$

where $\theta=C_{1} x+C_{2} t, C_{1}$ and $C_{2}$ are constants. Taking (5.21) into (5.20), one can get

$$
\begin{aligned}
& C_{2} v^{\prime} F+C_{2} v F^{\prime}+(\delta-d) C_{1}^{2} v^{\prime \prime} F+(\delta-2 d) C_{1}^{2} v^{\prime} F^{\prime}-d C_{1}^{2} v F^{\prime \prime}=0, \\
& C_{2} v^{\prime}-\alpha C_{1}^{2} v^{\prime \prime}-\beta v F+\lambda v=0 .
\end{aligned}
$$

When $\delta=d$, we can find a solution for (1.4) as follows:

$$
\begin{aligned}
F= & M_{1} e^{\frac{C_{2} \theta}{d C_{1}^{2}}}, \\
v= & M_{2} e^{\frac{C_{2} \theta}{2 \alpha C_{1}^{2}}} \operatorname{Bessel} \mathrm{J}\left(\frac{d \sqrt{C_{2}^{2}+4 \lambda \alpha C_{1}^{2}}}{C_{2} \alpha}, \frac{2 d C_{1} \sqrt{M_{1} \beta}}{C_{2} \sqrt{\alpha}} e^{\frac{C_{2} \theta}{2 d C_{1}^{2}}}\right) \\
& +M_{3} e^{\frac{C_{2} \theta}{2 \alpha C_{1}^{2}}} \operatorname{Bessel} \mathrm{Y}\left(\frac{d \sqrt{C_{2}^{2}+4 \lambda \alpha C_{1}^{2}}}{C_{2} \alpha}, \frac{2 d C_{1} \sqrt{M_{1} \beta}}{C_{2} \sqrt{\alpha}} e^{\frac{C_{2} \theta}{2 d C_{1}^{2}}}\right) .
\end{aligned}
$$


An exact solution of (1.4) can be derived from (5.19) and (5.23) as follows:

$$
\begin{aligned}
u= & M_{1} M_{2} e^{\frac{C_{2}(2 \alpha+d)\left(C_{1} x+C_{2} t\right)}{2 d \alpha C_{1}^{2}}} \operatorname{Bessel}\left(\frac{d \sqrt{C_{2}^{2}+4 \lambda \alpha C_{1}^{2}}}{C_{2} \alpha}, \frac{2 d C_{1} \sqrt{M_{1} \beta}}{C_{2} \sqrt{\alpha}} e^{\frac{C_{2}\left(C_{1} x+C_{2} t\right)}{2 d C_{1}^{2}}}\right) \\
& \left.+M_{1} M_{3} e^{\frac{C_{2}(2 \alpha+d)\left(C_{1} x+C_{2} t\right)}{2 d \alpha C_{1}^{2}}}\right) \\
& \times \operatorname{Bessel} \mathrm{Y}\left(\frac{d \sqrt{C_{2}^{2}+4 \lambda \alpha C_{1}^{2}}}{C_{2} \alpha}, \frac{2 d C_{1} \sqrt{M_{1} \beta}}{C_{2} \sqrt{\alpha}} e^{\frac{C_{2}\left(C_{1} x+C_{2} t\right)}{2 d C_{1}^{2}}}\right), \\
v= & M_{2} e^{\frac{C_{2}\left(C_{1} x+C_{2} t\right)}{2 \alpha C_{1}^{2}}} \operatorname{BesselJ}\left(\frac{d \sqrt{C_{2}^{2}+4 \lambda \alpha C_{1}^{2}}}{C_{2} \alpha}, \frac{2 d C_{1} \sqrt{M_{1} \beta}}{C_{2} \sqrt{\alpha}} e^{\frac{C_{2}\left(C_{1} x+C_{2} t\right)}{2 d C_{1}^{2}}}\right) \\
& +M_{3} e^{\frac{C_{2}\left(C_{1} x+C_{2} t\right)}{2 \alpha C_{1}^{2}}} \operatorname{BesselY}\left(\frac{d \sqrt{C_{2}^{2}+4 \lambda \alpha C_{1}^{2}}}{C_{2} \alpha}, \frac{2 d C_{1} \sqrt{M_{1} \beta}}{C_{2} \sqrt{\alpha}} e^{\frac{C_{2}\left(C_{1} x+C_{2} t\right)}{2 d C_{1}^{2}}}\right) .
\end{aligned}
$$

The two-dimensional and three-dimensional physical interpretations of solution (5.24) are presented in Figs. 1-4 by considering the values

$$
\begin{aligned}
& C_{1}=1, \quad C_{2}=1, \quad M_{1}=1, \quad M_{2}=1, \quad M_{3}=1, \\
& \alpha=1, \quad \beta=1, \quad d=1, \quad \lambda=\frac{15}{4} .
\end{aligned}
$$

From the symmetry $V_{4}=\frac{x}{2} \frac{\partial}{\partial x}+t \frac{\partial}{\partial t}-u \frac{\partial}{\partial u}$ for the special case $\lambda=0$, we can obtain

$$
\begin{aligned}
& u=\frac{1}{t} F, \\
& v=G,
\end{aligned}
$$

where $F=F(\theta), G=G(\theta), \theta=\frac{x^{2}}{t}$. Substituting (5.26) to (1.4), we can obtain the following reduction equations:

$$
\begin{aligned}
& -F-\theta F^{\prime}-4 d \theta F^{\prime \prime}-2 d F^{\prime}+2 \delta \frac{F G^{\prime}}{G}+4 \delta \theta\left(\frac{F G^{\prime}}{G}\right)^{\prime}=0, \\
& -\theta G^{\prime}-4 \alpha \theta G^{\prime \prime}-2 \alpha G^{\prime}-\beta F=0 .
\end{aligned}
$$

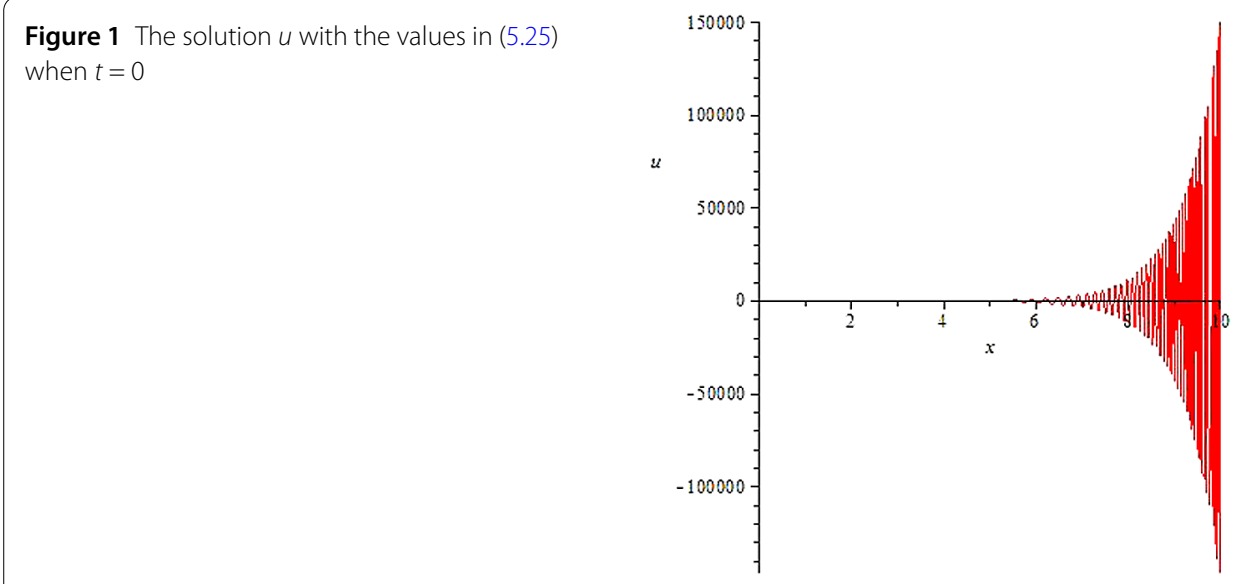


Figure 2 The solution $v$ with the values in (5.25) when $t=0$

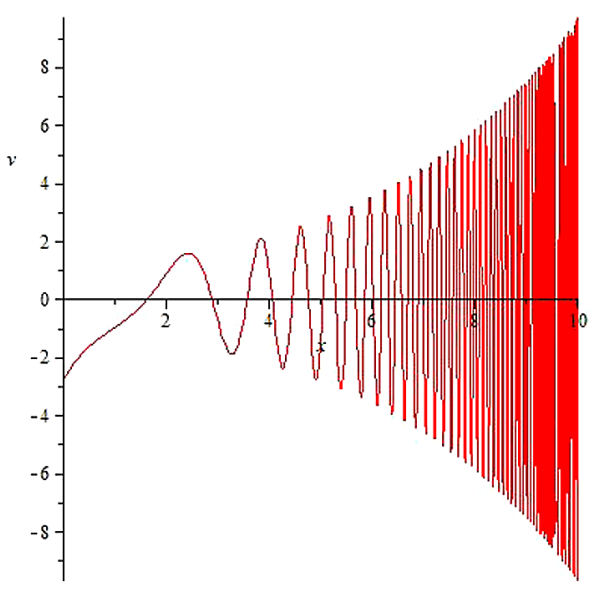

Figure 3 The solution $u$ with the values in (5.25)

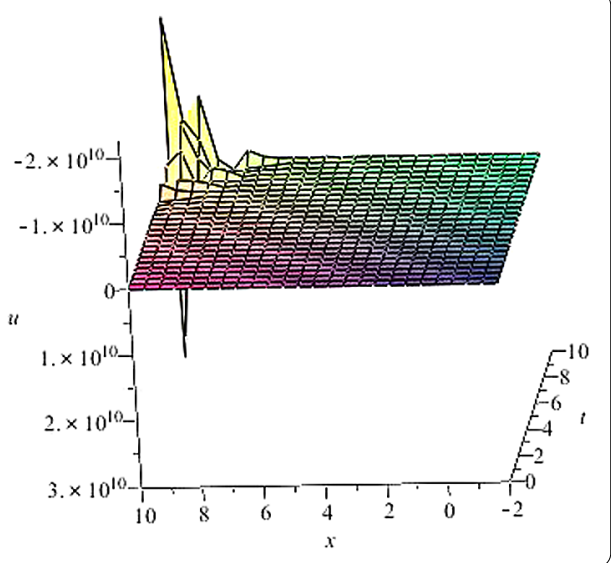

Figure 4 The solution $v$ with the values in (5.25)

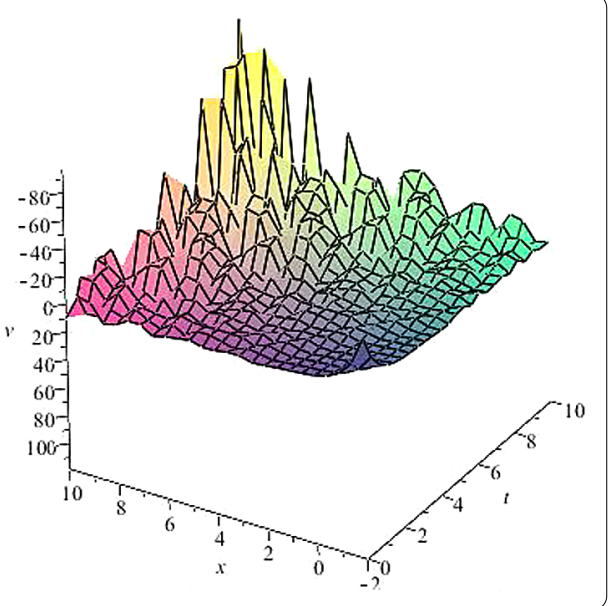


Remark 2 The solutions of reduction equations (5.5), (5.9), and (5.27) are difficult to find. It remains open to solve the three equations.

\section{Conclusions}

In summary, by performing Lie symmetry analysis on equations (1.2), (1.3), and (1.4), we obtain their Lie symmetries and find that their Lie symmetries are spanned by finite dimensional Lie algebra. According to the relationship between symmetry and conservation laws given by Ibragimov, many explicit and non-trivial conservation laws for the three equations are derived. These conservation laws may be useful for the explanation of some practical problems. Using the associated characteristic equation of the obtained symmetry, equations (1.2) and (1.4) are reduced to several ordinary differential equations. New explicit solutions of the two equations have been derived by solving the reduction equations. However, some of the reduction equations have not been solved analytically, the solutions of them will be our sustained concern in the future.

\section{Funding}

The work is supported by the Natural Science Foundation of Shandong Province (ZR2017LA012), Science and Technology Program of Colleges and Universities in Shandong (J17KA156), and the National Natural Science Foundation of China (11501082).

\section{Competing interests}

The authors declare that they have no competing interest.

\section{Authors' contributions}

The reduction equations were derived from symmetries and their solutions were obtained by the second author FX, the other part of the work is done by the author $L Z$. All authors read and approved the final manuscript.

\section{Publisher's Note}

Springer Nature remains neutral with regard to jurisdictional claims in published maps and institutional affiliations.

Received: 7 June 2018 Accepted: 20 July 2018 Published online: 18 September 2018

\section{References}

1. Keller, E.F., Segel, L.A.: Initiation of slime mold aggregation viewed as an instability. J. Theor. Biol. 26, 399-415 (1970)

2. Keller, E.F., Segel, L.A.: Model for chemotaxis. J. Theor. Biol. 30, 225-234 (1971)

3. Wang, Z.A.: Mathematics of traveling waves in chemotaxis. Discrete Contin. Dyn. Syst., Ser. B 18, 601-641 (2013)

4. Horstmann, D.: From 1970 until present: the Keller-Segel model in chemotaxis and its consequences, II. Jahresber. Dtsch. Math.-Ver. 106, 51-69 (2004)

5. Hillen, T., Painter, K.J.: A user's guide to PDE models for chemotaxis. J. Math. Biol. 58, 183-217 (2009)

6. Horstmann, D.: From 1970 until present: the Keller-Segel model in chemotaxis and its consequences, I. Jahresber. Dtsch. Math.-Ver. 105, 103-165 (2003)

7. Murray, J.: Mathematical Biology: II. Spatial Models and Biomedical Applications. Springer, New York (2003)

8. Shubina, M.: The 1D parabolic-parabolic Patlak-Keller-Segel model of chemotaxis: the particular integrable case and soliton solution. J. Math. Phys. 57, 091501 (2016)

9. Zhang, L.H., Ma, L.X., Xu, F.S.: New explicit exact solutions of the one-dimensional parabolic-parabolic Keller-Segel model. Appl. Comput. Math. 7, 50-57 (2018)

10. Keller, E.F., Segel, L.A.: Traveling bands of chemotactic bacteria: a theoretical analysis. J. Theor. Biol. 26, 235-248 (1971)

11. Adler, J.: Chemotaxis in bacteria. Science 153, 708-716 (1966)

12. Adler, J.: Chemoreceptors in bacteria. Science 166, 1588-1597 (1969)

13. Gerisch, A., Chaplain, M.A.J.: Mathematical modelling of cancer cell invasion of tissue: local and non-local models and the effect of adhesion. J. Theor. Biol. 250, 684-704 (2008)

14. Chaplain, M.A.J., Lachowicz, M., Szymanska, Z., Wrzosek, D.: Mathematical modelling of cancer invasion: the importance of cell-cell adhesion and cell-matrix adhesion. Math. Models Methods Appl. Sci. 21, 719-743 (2011)

15. Bluman, G.W., Anco, S.: Symmetry and Integration Methods for Differential Equations. Springer, New York (2002)

16. Ibragimov, N.H.: Transformation Groups Applied to Mathematical Physics. Reidel, Dordrecht (1985). Translated from the Russian Mathematics and Its Applications (Soviet Series)

17. Olver, P.J.: Applications of Lie Groups to Differential Equations. Springer, New York (1993)

18. Zhang, L.H.: Nonlinear self-adjointness and conservation laws of the variable coefficient combined KdV equation with a forced term. Adv. Differ. Equ. 2015, 229 (2015)

19. Buhe, E., Bluman, G.W.: Symmetry reductions, exact solutions, and conservation laws of the generalized Zakharov equations. J. Math. Phys. 56, 101501 (2015) 
20. Wang, G.W.: Symmetry analysis and rogue wave solutions for the $(2+1)$-dimensional nonlinear Schrödinger equation with variable coefficients. Appl. Math. Lett. 56, 56-64 (2016)

21. Liu, H.Z., Geng, Y.X.: Symmetry reductions and exact solutions to the systems of carbon nanotubes conveying fluid. J. Differ. Equ. 254, 2289-2303 (2013)

22. Baleanu, D., Inc, M., Yusuf, A., Aliyu, A...: Space-time fractional Rosenou-Haynam equation: Lie symmetry analysis, explicit solutions and conservation laws. Adv. Differ. Equ. 2018, 46 (2018)

23. Baleanu, D., Inc, M., Yusuf, A., Aliyu, A.I.: Time fractional third-order evolution equation: symmetry analysis, explicit solutions, and conservation laws. J. Comput. Nonlinear Dyn. 13, 021011 (2018)

24. Baleanu, D., Inc, M., Yusuf, A., Aliyu, A.I.: Lie symmetry analysis, explicit solutions and conservation laws for the space-time fractional nonlinear evolution equations. Physica A 496, 371-383 (2018)

25. Baleanu, D., Inc, M., Yusuf, A., Aliyu, A.I.: Lie symmetry analysis, exact solutions and conservation laws for the time fractional Caudrey-Dodd-Gibbon-Sawada-Kotera equation. Commun. Nonlinear Sci. Numer. Simul. 59, 222-234 (2018)

26. Roman, C., Maksym, D.: Exact solutions of the simplified Keller-Segel model. Commun. Nonlinear Sci. Numer. Simul. 18, 2960-2971 (2013)

27. Maksym, D.: A (1 + 2)-dimensional simplified Keller-Segel model: Lie symmetry and exact solutions. Symmetry 7 , 1463-1474 (2015)

28. Roman, C., Maksym, D.: A (1 + 2)-dimensional simplified Keller-Segel model: Lie symmetry and exact solutions, II. Symmetry 9, 1-17 (2017)

29. Lax, P.D.: Integrals of nonlinear equations of evolution and solitary waves. Commun. Pure Appl. Math. 21, 467-490 (1968)

30. Knops, R.J., Stuart, C.A.: Quasi convexity and uniqueness of equilibrium solutions in nonlinear elasticity. Arch. Ration Mech. Anal. 86, 234-249 (1984)

31. Benjamin, T.B.: The stability of solitary waves. Proc. R. Soc. Lond. A 328, 153-183 (1972)

32. Noether, E.: Invariante variations probleme. Nachr. König. Gesel. Wissen. Göttingen. Math. Phys. Kl. Heft. 2, 235-257 (1918)

33. Kara, A.H., Mahomed, F.M.: Noether-type symmetries and conservation laws via partial Lagrangians. Nonlinear Dyn. 45, 367-383 (2006)

34. Ibragimov, N.H.: A new conservation theorem. J. Math. Anal. Appl. 333, 311-328 (2007)

35. Baleanu, D., Inc, M., Yusuf, A., Aliyu, A.l.: Optical solitons, nonlinear self-adjointness and conservation laws for Kundu-Eckhaus equation. Chin. J. Phys. 55, 2341-2355 (2017)

36. Inc, M., Aliyu, A.I., Yusuf, A.: Solitons and conservation laws to the resonance nonlinear Schrodinger's equation with both spatio-temporal and inter-modal dispersion. Optik 142, 509-522 (2017)

37. Baleanu, D., Inc, M., Yusuf, A., Aliyu, A.I.: Travelling wave solutions and conservation laws for nonlinear evolution equation. J. Math. Phys. 59, 023506 (2018)

38. Inc, M., Yusuf, A., Aliyu, A.I., Hashemi, M.S.: Soliton solutions, stability analysis and conservation laws for the Brusselator reaction diffusion model with time and constant dependent coefficients. Eur. Phys. J. Plus 133, 168 (2018)

39. Ibragimov, N.H.: Integrating factors, adjoint equations and Lagrangians. J. Math. Anal. Appl. 318, $742-757$ (2006)

40. Buhe, E., Bluman, G.W., Kara, A.H.: Conservation laws for some systems of nonlinear PDEs via the symmetry/adjoint symmetry pair method. J. Math. Anal. Appl. 436, 94-103 (2016)

41. Anco, S.C., Bluman, G.W.: Direct construction of conservation laws from field equations. Phys. Rev. Lett. 78, 2869-2873 (1997)

\section{Submit your manuscript to a SpringerOpen ${ }^{\circ}$ journal and benefit from:}

- Convenient online submission

- Rigorous peer review

- Open access: articles freely available online

- High visibility within the field

- Retaining the copyright to your article

Submit your next manuscript at $\gg$ springeropen.com 